\title{
Predictive Energy Consumption in Intelligent Buildings
}

\author{
Iulian Dorin SBANCA \\ Transilvania University of Brasov, Romania, dorinel.sbanca@unitbv.ro \\ Mariana FRATU \\ Transilvania University of Brasov, Romania, mariana.fratu@unitbv.ro
}

\begin{abstract}
In this work we present a novel approach to energy saving in buildings through the identification of the relevant parameters and the application of Soft Computing techniques to generate predictive models of energy consumption in buildings. To verify the feasibility of this proposal, we apply our approach to a reference building for which we have contextual data from a complete year of monitoring. First, we characterize the building in terms of its contextual features and energy consumption, and then select the most appropriate techniques to generate the most accurate model of our reference building charged with estimating the energy consumption, given a concrete set of inputs.
\end{abstract}

\section{Keywords}

big data, smart buildings, energy consumption, energy efficiency

\section{Introduction}

In recent time different approaches have addressed the energy efficiency of buildings using predictive models of energy consumption based on usage profile, climate data and building characteristics. The integration and development of systems based on Information and Communication Technologies (ICT), and more specially, the Internet of Things (IoT) are important enablers of a wide range of applications, for the general population, helping make intelligent buildings a reality.

The IoT has provided vast amounts of data that can be analyzed deeply to reveal interesting relationships, which can be used to generate models able to anticipate and respond efficiently to certain events. Big data and IoT are a perfect combination that can be applied to Smart Buildings scenarios for energy efficiency [1]. The approach of this paper involves applying insights from Big data algorithms to sensed data in intelligent buildings.

Our approach address to energy saving and energy efficiency in buildings based on using the data measured by sensors installed in the building. For the extraction of relevant knowledge from all the sensed data to model the energy consumption profile of buildings, we apply sophisticated Soft Computing techniques. Once energy usage profiles have been extracted, we can design and implement actions to save energy. For the generation of predictive models, we use the data collected during a monitored period. Then, considering such models, we propose some measurements of control to save energy in the building taken as reference.

\section{Related Work}

Initial solutions were mainly focused on using non-deterministic models based on simulations. A number of simulation tools are available with varying capabilities. In [2] a comprehensive comparison of existing simulation tools is provided. This type of approach relies on predictive models based on static perceptions of the environment.

For example, a multi-criteria decision model to evaluate the whole life cycle of a building is presented in [3]. The authors deal with the problem from a multi-objective optimization viewpoint and conclude that finding an optimal solution is unreal, and that only an approximation is feasible. Timers and motion sensors provide a useful tool to detect and respond to occupants, while providing them with feedback information based on real sensor data and contextual information [4].

The approach of this paper involves predictive models based on a combination of real data and predictive patterns that represent the evolution of the parameters affecting energy consumption in 
buildings. We propose to generate accurate predictive models of energy building consumption to be used to select the best strategies for saving energy of the specific characteristics of each building.

\section{Optimum Strategies for Energy Efficiency in Intelligent Buildings}

The intelligent buildings must be capable of not only providing mechanisms to minimize their energy consumption (even integrating their own energy sources to ensure their energy sustainability), but also improving occupant experience and activity efficiency. In recent time different approaches have addressed the energy efficiency of buildings using predictive models of energy consumption based on usage profile, climate data and building characteristics.

Optimizing energy efficiency in buildings is an integrated task that covers the whole life cycle of the building. Herein after, we refer only to electrical energy consumption since other kinds of energy such as fuel oil, gas or water are beyond the scope of this work.

Therefore, it is first necessary to identify the main drivers of energy use in buildings. After monitoring these parameters, we can model their impact on energy consumption.

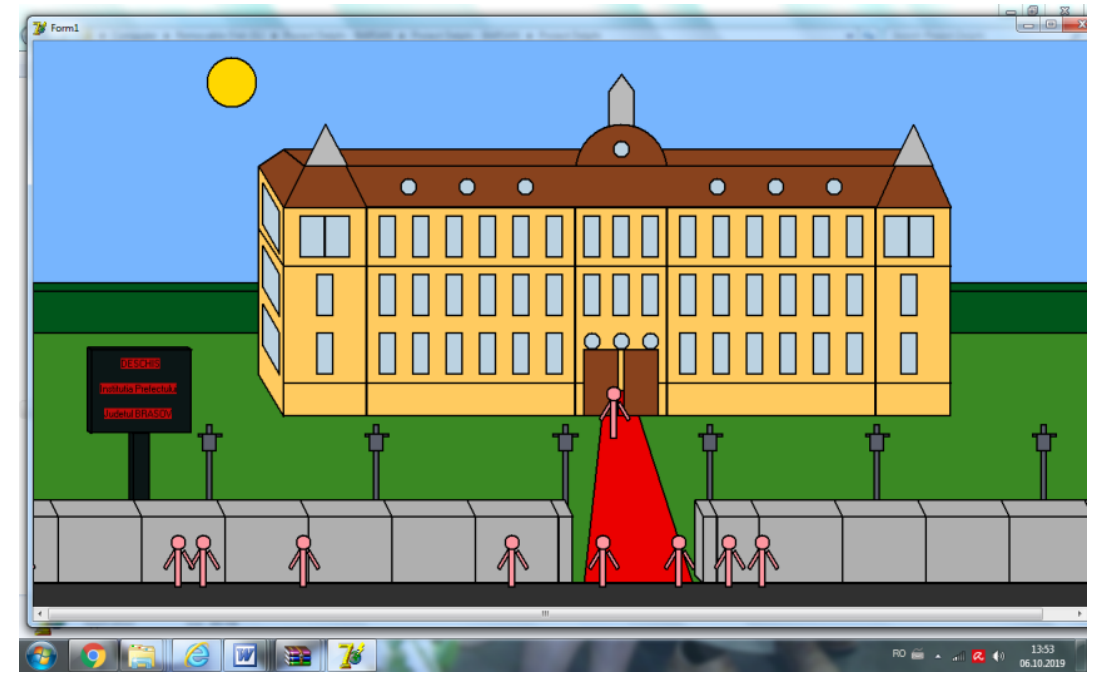

Fig. 1. Virtual model of the reference public building

In this building, the first stages of experimentation have been already carried out following the approach proposed in this work. Analysis of the generated models has led to energy saving strategies being applied.

First, we analyze what the main drivers of the energy consumed in buildings are. For this analysis, we use the data measured by sensors installed in the building and thus generate the predictive model that estimates its daily energy consumption.

As a real case where energy saving must be achieved, we present a polyvalent public building with high levels of monthly energy consumption involved in thermal comfort provision.

Considering this, and taking into account the models for predicting the comfort response of building's occupants, we describe below the main parameters that must be monitored and analyzed before selecting optimum strategies to save energy.

1. Electrical devices always connected to the electrical network. In buildings, it is necessary to characterize the minimum value of energy consumption due to electrical devices that are always connected to the electrical network.

2. Electrical devices occasionally connected to the electrical network. Depending on the kind of building under analysis, different electrical devices must be included in the final system responsible for estimating the daily electrical consumption of the building.

3. Occupants' behavior. Energy consumption of buildings due to the behavior of their occupants is one of the most critical points in every building energy management system.

4. Environmental conditions Parameters such as temperature, humidity, pressure, and natural lighting have a direct impact on the energy consumption of buildings. 
5. Information about the energy generated in the building. Information about the amount of daily energy generated and its associated contextual features can be used to estimate the total energy generated in the future.

6. Information about energy consumed in the building. From this set of parameters affecting energy consumption, we can extract the input data to be included in the estimation of the target building energy consumption model.

Based on all these parameters, it is possible to design optimum strategies to save energy. Therefore, the approach proposed to design optimum strategies of energy saving in buildings is the following:

a) Analyze the energy consumption profile associated to each service provided in the building. In this way, it is possible to identify variables affecting the energy consumption of each service.

b) Analyze the relation among the evolution of such variables and the energy consumed. Thus, it is possible to select variables with the most relevant impact in the energy consumption.

c) Provide behavior patterns of the variables identified as relevant, including their uncertainty

d) Implement a predictive building model able to estimate the evolution of the energy consumption given such a set of inputs.

e) Design optimum strategies of control to save energy in the building based on the estimated evolution of the energy consumption.

We propose a procedure based on applying different soft computing techniques according to the specific goal to be achieved. After carrying out these steps, an estimator is able to predict the energy consumption in an on-line way.

\section{Generating the Energy Consumption Models of the Reference Building}

Due to the features of our reference building, we focus on modeling its energy consumption associated to the time periods in which the building is occupied. We describe the computational techniques as follow:

1. Data collection. During this first stage, we collect data about outdoor/indoor temperature, humidity and pressure. The Energy Consumption (EC) is collected over short periods of time (each minute of every day during a year).

Such measurements are associated to specific vectors of environmental parameters (Z(t)) measured outside and inside the building. Thus, the building models generated will be sufficiently representative to cover different contextual conditions.

So, the set of data pairs for the training of our building model is:

$$
(\mathrm{EC}(t), \mathrm{Z}(t)), t=1,2, \ldots, \mathrm{N},
$$

where $\mathrm{N}$ is the number of data instances collected during $1 \mathrm{~h}$ of monitoring.

Electrical Energy Consumption, $\mathrm{EC}(t)$ refer to the environmental parameters vector associated to the energy consumption measured at the instant $t$.

2. Pre-processing. The pre-processing unit is responsible for transforming the measured data. The different processing techniques applied in this stage are given as follows:

- Transformation based on the raw dataset collected. During the transformation, compact representations of the input data, namely features, are extracted, which will be used later for energy consumption estimation.

- Filtering. During this process a filter is applied that removes features extracted from the training data set that does not vary at all or that varies too much.

- Normalization. All values in the given dataset are normalized during this phase. The resulting values are in the $[0,1]$ interval for every feature extracted from the initial dataset.

- Feature selection. We apply Principal Component Analysis (PCA) in conjunction with a ranker search mechanism.

With the aim of reducing the final computational load of the estimation method, we searched the optimum number of attributes to represent the energy consumption profile of our reference building. After this analysis, we found that outdoor temperature, humidity and pressure were the features selected by the ranked feature combination technique used by the PCA. 
PCA mechanism is a statistical procedure that uses an orthogonal transformation to convert a set of observations of possibly correlated variables into a set of values of linearly uncorrelated variables called principal components. This transformation is defined in such a way that the first principal component has the largest possible variance and each succeeding component has the highest variance possible under the constraint. In our approach the number of features was reduced to 3 , which will be denoted as $\mathrm{f}_{1}, \mathrm{f}_{2}, \mathrm{f}_{3}$.

Considering this vector of features, Eq. (1) can be rewritten as:

$$
\left\{\left[\mathrm{f}_{1}(t), \mathrm{f}_{2}(t), \mathrm{f}_{3}(t)\right], \mathrm{Z}(t)\right\}, t=1,2, \ldots, \mathrm{N} \text {. }
$$

At this point, we generate the maps of the building based on the selected features.

3. Clustering. During this stage, the input data division according to the distribution of the values of these features is carried out, the data being grouped according to the identified clusters, whose centroids are associated to landmarks.

4. Landmark classifier. After classifying the energy consumption landmark for each new measurement, we can focus on the outdoor temperature characterization of such landmark.

5. Energy consumption estimator. For consumption estimation, the interpolation algorithm Radial Basis Functions (RBF) network for each landmark is computed as regression technique.

RBF network uses all training data associated to every landmark. The input space P of our RBF, is the vector of the mean values of the outdoor environmental parameters. These data can be denoted as:

$$
\mathrm{P}=\left\{\mathrm{p}_{\mathrm{i}}\right\}, \forall \mathrm{p}_{\mathrm{i}}=\left[\mathrm{p}_{1}, \mathrm{p}_{2}, \ldots, \mathrm{p}_{\mathrm{n}}\right],
$$

where $\mathrm{n}$ is the number of measurements gathered and classified within the chosen subset associated to a landmark. The target class $\mathrm{Z}\left(\mathrm{Z} \in \mathrm{R}_{\mathrm{k}}\right)$ represents the energy consumption. This is denoted as:

$$
\mathrm{Z}=\left\{\mathrm{Z}_{\mathrm{ik}}\right\}, \forall \mathrm{z}_{\mathrm{ki}}=\left[\mathrm{Z}_{\mathrm{k} 1}, \mathrm{Z}_{\mathrm{k} 2}, \ldots, \mathrm{z}_{\mathrm{kn}}\right] .
$$

Then, given the training values $\left\{\left(\mathrm{p}_{\mathrm{i}}, \mathrm{z}_{\mathrm{ki}}\right), \ldots,\left(\mathrm{p}_{\mathrm{n}}, \mathrm{z}_{\mathrm{kn}}\right)\right\}$, our goal is to find a function that will allow us to classify the monitored energy consumption $\left(z_{i}\right)$, giving its vector of features $\left(p_{i}=\left[f_{1 i}, f_{2 i}, f_{3 i}\right]\right)$. The vector $p_{j}$ is provided as input to all functions of our RBF.

\section{Conclusion}

With the continual progress made in the field of ICT and sensor networks, new applications based on using extensive number of different sensors to monitor building environments are being proposed to improve energy efficiency of buildings through the integration of massive volume of data.

Our approach address to energy saving and energy efficiency in buildings based on using the data measured by sensors installed in the building. For the extraction of relevant knowledge from all the sensed data to model the energy consumption profile of buildings, we apply sophisticated Soft Computing techniques.

At present we are carrying out experiments to analyze the impact of implementing the strategies proposed to save energy in the building under experimentation $[5,6]$.

\section{References}

1. Moreno M.V., et al. (2016): Big Data, the Key to Energy Efficiency in Smart Buildings. Soft Computing, ISSN 1432-7643, vol. 20, is. 5, p. 1749-1762, https://hal.archives-ouvertes.fr/hal-01620293/document

2. Patel S., et al. (2007): At the Flick of a Switch: Detecting and Classifying Unique Electrical Events on the Residential Power Line. In: J. Krumm et al. (Eds.): UbiComp 2007, LNCS 4717, Springer Berlin Heidelberg, p. 271-288, https://homes.cs.washington.edu/ shwetak/papers/ubicomp2007 flick.pdf

3. Scott J., et al. (2011): Preheat: Controlling Home Heating Using Occupancy Prediction. Proc. of the 13th int. conf. on Ubiquitous computing (UbiComp '11), ACM, p. 281-290, https://doi.org/10.1145/2030112.2030151

4. Lu J., et al. (2010): The Smart Thermostat: Using Occupancy Sensors to Save Energy in Homes. The 8th ACM Conference SenSys 2010, p. 211-224, http://sensys.acm.org/2010/Papers/p211-Lu.pdf

5. https://ec.europa.eu/info/news/new-energy-performance-buildings-directive-comes-force-9-july-2018-018jun-19 en

6. https://eur-lex.europa.eu/legal-content/EN/TXT/?toc=0J\%3AL $\% 3$ A2018\%3A156\%3ATOC\&uri =uriserv $\% 3$ AOJ.L.2018.156.01.0075.01.ENG 